\title{
Ibrutinib induces chromatin reorganisation of chronic lymphocytic leukaemia cells
}

\author{
Katie B. Holmes ${ }^{1}$, Ildar I. Sadreev ${ }^{1,2}$, Andy C. Rawstron $\mathbb{1}^{3}$, Tal Munir ${ }^{3}$, David R. Westhead ${ }^{2}$, Peter Hillmen ${ }^{1}$ and \\ Pascal F. Lefevre (iD ${ }^{1}$
}

\begin{abstract}
Chronic lymphocytic leukaemia (CLL) is the most common leukaemia in Western countries. It has recently been shown that the homogeneity of the chromatin landscape between CLL cells contrasts with the important observed genetic heterogeneity of the disease. To gain further insight into the consequences of disease evolution on the epigenome's plasticity, we monitored changes in chromatin structure occurring in vivo in CLL cells from patients receiving continuous Ibrutinib treatment. Ibrutinib, an oral inhibitor of the Bruton's tyrosine kinase (BTK) has proved to be remarkably efficient against treatment naïve (TN), heavily pre-treated and high-risk chronic lymphocytic leukaemia (CLL), with limited adverse events. We established that the chromatin landscape is significantly and globally affected in response to Ibrutinib. However, we observed that prior to treatment, CLL cells show qualitative and quantitative variations in chromatin structure correlated with both $\mathrm{EZH} 2$ protein level and cellular response to external stimuli. Then, under prolonged exposure to Ibrutinib, a loss of the two marks associated with lysine 27 (acetylation and trimethylation) was observed. Altogether, these data indicate that the epigenome of CLL cells from the peripheral blood change dynamically in response to stimuli and suggest that these cells might adapt to the Ibrutinib "hit" in a process leading toward a possible reduced sensitivity to treatment.
\end{abstract}

\section{Introduction}

Chronic lymphocytic leukaemia (CLL) originates from clonal proliferating B-cells with patients mainly presenting with lymphadenopathy, splenomegaly, and lymphocytosis ${ }^{1}$. A combination of fludarabine, cyclophosphamide and rituximab (FCR) represents the standard therapy ${ }^{2}$. However, a majority of patients relapse with most of them eventually succumbing to CLL. Encouraging results of several forerunner clinical trials that target the activity of PI3K $\delta$, BTK or SYK, highlight the therapeutic potential of inhibiting BCR signalling ${ }^{3,4}$. Ibrutinib (PCI-32765), a

\footnotetext{
Correspondence: Pascal F. Lefevre (p.lefevre@leeds.ac.uk)

${ }^{1}$ Section of Experimental Haematology, Leeds Institute of Medical Research at St James's, University of Leeds, Leeds, UK

'Bioinformatics Group, Institute of Molecular and Cellular Biology, University of Leeds, Leeds, UK

Full list of author information is available at the end of the article.

These authors contributed equally: Katie B. Holmes, Ildar I. Sadreev
}

specific and irreversible inhibitor of Bruton's Tyrosine Kinase (BTK), is a small molecule orally administered, providing a selective and irreversible inhibition of BTK. In extensive studies, Ibrutinib has shown extremely promising results in front-line treatment as well as in relapsed/refractory (RR) $\mathrm{CLLs}^{5,6}$ and is now tested in combination with other molecules ${ }^{7}$. However, cases of drug resistance have emerged ${ }^{8,9}$.

In recent years, a large body of work has highlighted the complexity of the regulatory mechanisms controlling gene expression by external environmental stimuli and signalling pathways for which chromatin plays a central role. The eukaryotic genomes are partitioned into functionally autonomous clusters in which gene expression is either positively or negatively controlled. In active clusters, promoters are highly enriched for the histone lysine 4 trimethylation mark (H3K4me3), whereas activated enhancers display enrichment of histone $\mathrm{H} 3$ lysine 4 mono-methylation and di-methylation (H3K4me1/2) and

\section{(c) The Author(s) 2019}

(c) Open Access This article is licensed under a Creative Commons Attribution 4.0 International License, which permits use, sharing, adaptation, distribution and reproduction cc) in any medium or format, as long as you give appropriate credit to the original author(s) and the source, provide a link to the Creative Commons license, and indicate if changes were made. The images or other third party material in this article are included in the article's Creative Commons license, unless indicated otherwise in a credit line to the material. If material is not included in the article's Creative Commons license and your intended use is not permitted by statutory regulation or exceeds the permitted use, you will need to obtain permission directly from the copyright holder. To view a copy of this license, visit http://creativecommons.org/licenses/by/4.0/. 
lysine 27 acetylation (H3K27ac). The equilibrium between open and repressed chromatin is dynamic and can change transiently or permanently in response to various endogenous or exogenous stimuli. These processes are controlled by several classes of epigenetic factors. One such class of key epigenetic regulators are the polycomb group (PcG) proteins, which are a family of transcriptional repressors, primarily known in maintaining cell identity, but also implicated in the control of cellular proliferation and neoplastic development ${ }^{10-12}$. A recent study has shown that the lack of transcription triggers deposition of $\mathrm{H} 3 \mathrm{~K} 27 \mathrm{me} 3$, the repressive mark mediated by the polycomb-repressive complex $2(\mathrm{PRC} 2)^{13}$. The core PRC2 complex comprises of four components, its enzymatic subunit with methyltransferase activity EZH1 or EZH2, SUZ12, EED and RbAp46/48. Furthermore, bivalent promoters, which harbour both active and silent marks (H3K4me3, H3K27me3), are usually CpG rich $^{14}$. They have been mainly identified in stem cells, but can persist during differentiation as seen in $\mathrm{T}$ and $\mathrm{B}$ cells ${ }^{15}$.

EZH2 expression is correlated with proliferation to oppose cell division-mediated dilution of $\mathrm{H} 3 \mathrm{~K} 27 \mathrm{me} 3^{16}$. In B cells, EZH2 is highly expressed in lymphoid progenitors and is necessary for early lymphopoiesis ${ }^{17}$. EZH2 declines in mature resting $B$ cells but is transiently reactivated in the germinal centre where dividing Ki67+ centroblasts are associated with its expression ${ }^{18,19}$. EZH2 is required for the formation and function of the germinal centre, where it participates to the establishment of bivalency at key regulatory promoters to transiently block differentiation ${ }^{15}$.

A recent study proposed an extensive epigenomic characterisation of CLL cells, which indicated that if DNA methylation or chromatin accessibility shows patterns characteristic of the cellular origin of these cells, active chromatin marks like H3K27ac follow other more complex dynamics ${ }^{20}$. To further assess the correlation between chromatin organisation and the evolution of the disease, we analysed the plasticity of the chromatin landscape of CLL cells from patients treated with Ibrutinib.

Our analysis revealed that the CLL cell populations in the peripheral blood was heterogeneous, including cells with various proportions of epigenomic traits characteristic of activated B cells. Moreover, the initial chromatin remodelling in response to Ibrutinib was dependent upon these initial traits but was converging toward a similar organisation after few months. Prolonged exposure to Ibrutinib, inducing forced maintenance of cells in G0 phase, was associated with a complete loss of H3K27 posttranslational modifications including the polycombdependent repressive mark. This work suggests that CLL cells lose some epigenetic constraints in response to treatment.

\section{Results}

\section{Prolonged Ibrutinib treatment correlates with loss of histone H3K27 marks}

The IcICLLe trial is a feasibility study investigating the mechanism of action of Ibrutinib. Patients received continuous oral therapy with Ibrutinib (420 mg OD) and CLL cells were collected from the peripheral blood before and at regular time points during treatment (Supplementary Table 1). The percentage of Ki67-positive cells in the blood increased in the first $24 \mathrm{~h}$ after Ibrutinib treatment initiation but then dropped rapidly to completely disappear after 14 days in all patients (Fig. 1a). At the same time, an important and transient increase in CLL cell count, characteristic of Ibrutinib-dependent lymphocytosis, occurred within the first hours after the start of the treatment and fall back significantly to its initial level from 14 days and 2 months for treatment naïve (TN) and RR patients, respectively (Fig. 1b). This result is consistent with previously described Ibrutinib-dependent rapid reduction of lymphadenopathy accompanied by transient lymphocytosis in $\mathrm{CLL}^{21}$.

EZH2 is a marker of proliferation transiently reactivated in proliferation centre in normal B cells ${ }^{16,18,19}$. Therefore, we hypothesised that EZH2 protein level could serve as a suitable marker of lymphocytosis. EZH2 expression was not correlated with the evolution of B cell count in the PB, but either transiently increased in CLL after 14-28 days on Ibrutinib or present at a moderate level before treatment and going progressively down with prolonged exposure to the drug (Fig. 2a). Then, ChIP-seq experiments were performed for EZH2, H3K4me3 and H3K27me3 at 0, 7 and 56 days after the first treatment administration for $\mathrm{N} 1$ and N2 patients and H3K27ac for patients N3 and R3 (Fig. 2, Supplementary Fig. S1). Within H3K27me3-low regions, H3K27ac and/or H3K4me3 peaks corresponding to clusters of transcribing genes were observed as seen for the CXCR4 locus and two genomic regions randomly chosen and centred on the FOXP1 and BCL2 genes, respectively (Fig. 2b, Supplementary Fig. S1). In patient N2, in response to Ibrutinib and correlated with lymphocytosis, we observed, at 7 days, a transient and genome-wide increase in EZH2 recruitment and H3K27me3 at "active" regions (Fig. 2b, Supplementary Fig. S1B, D). This transient increase in H3K27me3 was also detected by conventional ChIP in six randomly chosen patients from the trial as shown for patient N4 (Fig. 2c). In contrast, in patient N1, EZH2/ H3K27me3 enrichment within "active" regions was seen at the time of treatment initiation (Fig. 2b, Supplementary Fig. S1A and C). For this patient, the enhanced level of H3K27me3 correlating with EZH2 recruitment was subtler than when observed post treatment.

In addition, independently of the initial EZH2 protein level, prolonged and continuous exposure to Ibrutinib- 

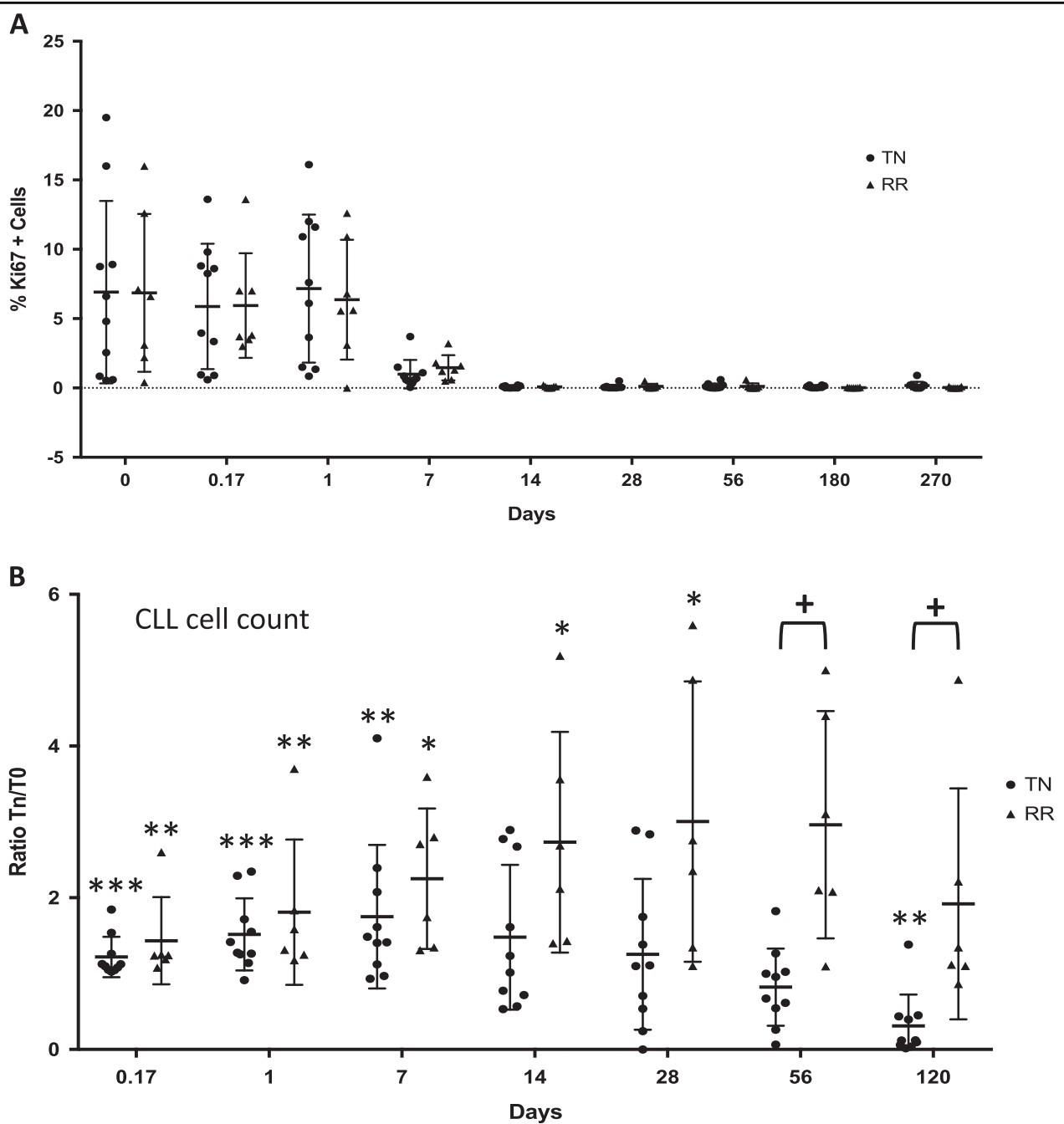

Fig. 1 lbrutinib treatment induces lymphocytosis in CLL cells. a Evolution of CLL cells count and (B) Ki67+ cells for the 16 patients included in the study comparing treatment-naïve (TN) and relapsed-refractory (RR) patients during (a) the first 120 days or (b) up to 9 months on lbrutinib. Peripheral CLL cell counts from peripheral blood are expressed as a ratio vs. cell count the day of the first Ibrutinib ingestion. Paired Student's $t$-test has been performed comparing cell count at variable time points compared to T0, ${ }^{*} t$-value $<0.05$, ${ }^{* *} t$-value $<0.01$ and ${ }^{* * *} t$-value $<0.001$. Student's $t$ test has also been performed comparing TN to RR for each time point, ${ }^{+} t$-value $<0.05$

induced loss of EZH2 protein and H3K27me3 (Fig. 2a, d, Supplementary Fig. S2B). Similarly, H3K27ac, which antagonises H3K27me3, disappeared from 56 days after treatment initiation in contrast to the H3K4me3 mark which was relatively stable upon prolonged drug exposure (Fig. 2b, d, Supplementary Fig. S2A). In conclusion, these data show that long-term Ibrutinib treatment induced a severe reduction of both histone $\mathrm{H} 3$ lysine 27 acetylation and methylation together with EZH2 protein disappearance in CLL cells.

\section{In depth analysis of EZH2 recruitment to active cis- regulatory elements}

To study EZH2 recruitment and H3K27me3 deposition in "active" chromatin domain in more detail, we separated the cis-regulatory elements positive for EZH2, H3K4me3 and/or H3K27me3 into seven subgroups before treatment and after 7 days on Ibrutinib. This analysis was performed with the data obtained from patient N1 (Fig. 3a, b) as the remarkable genome-wide increase of $\mathrm{H} 3 \mathrm{~K} 27 \mathrm{me} 3$ in patient $\mathrm{N} 2$ at 7 days was preventing an accurate quantification of positive peaks vs. background.

When EZH2 was targeting regions enriched for $\mathrm{H} 3 \mathrm{~K} 27 \mathrm{me} 3$, we observed only $2.5 \%$ increase in EZH2 bound cis-elements between 0 and 7 days (subgroups 1 and 2) (Fig. 3a, b). In contrast, if EZH2-targeted regions were negative for $\mathrm{H} 3 \mathrm{~K} 27 \mathrm{me} 3, \mathrm{EZH} 2$ occupancy decreased by $38 \%$ (EZH2-only, subgroup 4) and 50\% (EZH2 and H3K4me3, subgroup 3) after 7 days on Ibrutinib. An opposite evolution ( $30 \%$ increase) was observed for 


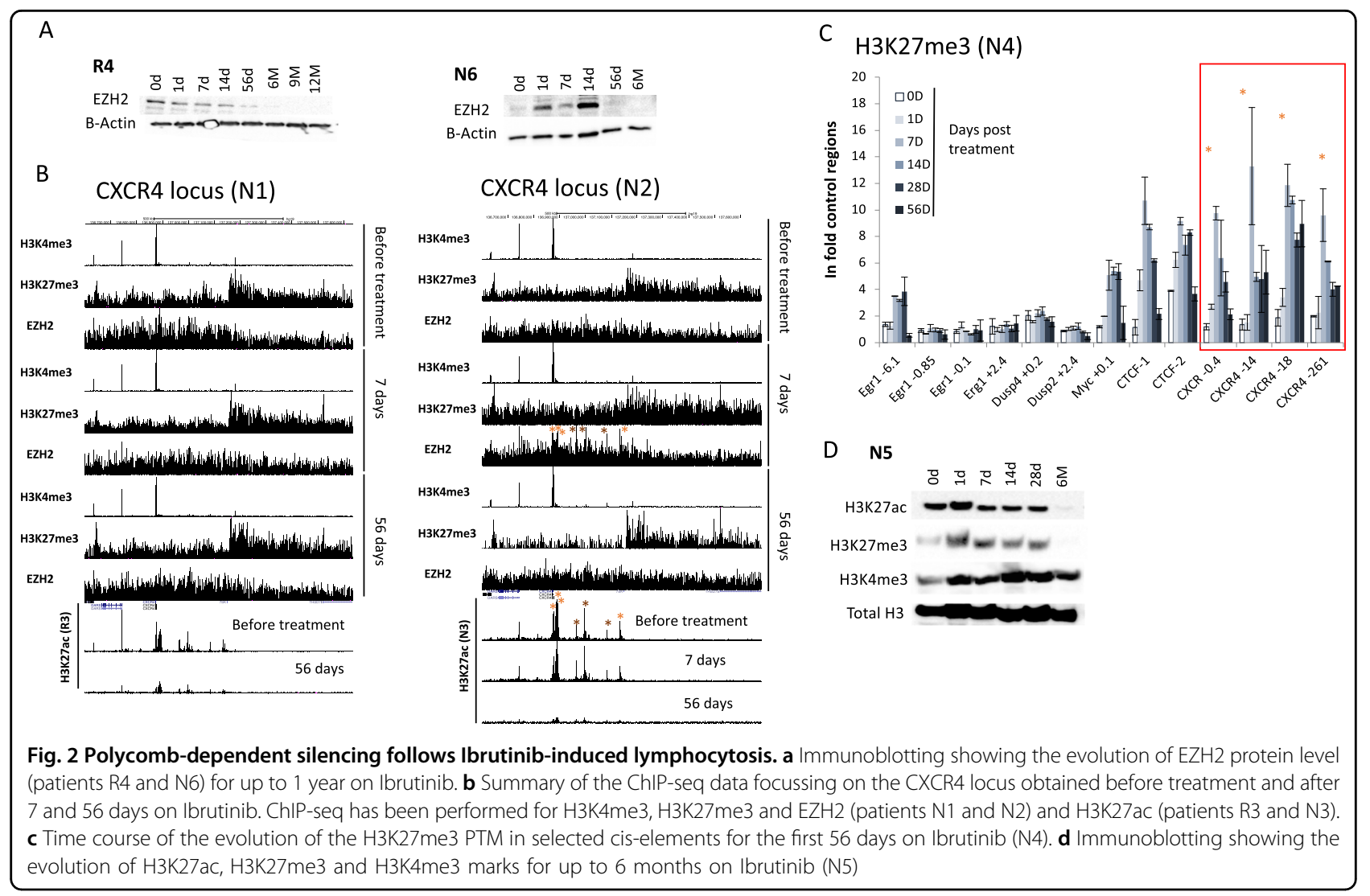

H3K4me3-only regions (subgroup 7) (Fig. 3a, b). Moreover, EZH2 was redeployed from H3K4me3 high promoters to H3K27me3-enriched region as highlighted with EZH2 barcodes (subgroups 2 and 4) (Fig. 3b). Altogether, these data indicated that the mechanism of EZH2 dissociation from active clusters described above was partially due to a redeployment of EZH2 to repressed chromatin in addition to a global decrease in EZH2 protein associated with chromatin.

Next, we analysed the intersection between our peak libraries and merged transcription factors ChIP-seq data obtained from a publicly available database (ReMap). This analysis, referred to as ReMap and Epigenetic Marks Intersection (REMI), calculates the number of cisregulatory elements enriched for a specific histone mark and containing a validated consensus sequence corresponding to a specific transcription factor. It allowed us to generate "signatures" associated with determined peak populations and to assess the relative variation of these signatures when comparing one peak population to another (Supplementary Figs. S3 and 4). For example, we established that non-promoter elements were enriched for transcription factors expressed in B cells and important for B cell function including SMAD1, IKZF1, BATF or $\mathrm{BCL} 11 \mathrm{~A}^{22-25}$, and promoter elements enriched for proteins like KDM5A/B, IRF3, TAF3 and GTF2B (Supplementary Figs. S3 and 4).

Using REMI analysis, we observed that before treatment, $\mathrm{H} 3 \mathrm{~K} 4 \mathrm{me} 3^{+} / \mathrm{EZH}^{+}{ }^{+}$cis-elements were enriched for factors detected at promoters and depleted from specific categories of peaks enriched for SMAD2/3/4, SOX2 and EZH2/SUZ12 (Supplementary Fig. S5A-D). It was at first surprising to find EZH2-bound regions depleted from the EZH2/SUZ12 validated sequences assembled in the ReMap database. However, when analysing the repartition of EZH2-assembled sequences from ReMap compared to the seven subgroups identified in Fig. 3, we determined that these sequences were mainly associated with bivalent promoters (Supplementary Fig. S5B). Moreover, these sequences were also the most represented at $\mathrm{CpG}$ islands when compared to promoter regions (Supplementary Fig. S6B). We concluded that our ChIP-seq experiments were showing an under-representation of EZH2 in regions where chromatin was "permanently" repressed (H3K27me3 was stable with time on Ibrutinib). These regions are enriched for the EZH2-validated sequences from ReMap (e.g. ChIP-seq performed on stem cells or partially dedifferentiated cell lines).

SMAD2, 3 and 4 were also found depleted from the $\mathrm{H} 3 \mathrm{~K}_{4} \mathrm{me}^{+} / \mathrm{EZH}^{+}$fraction. Most of SMADs 


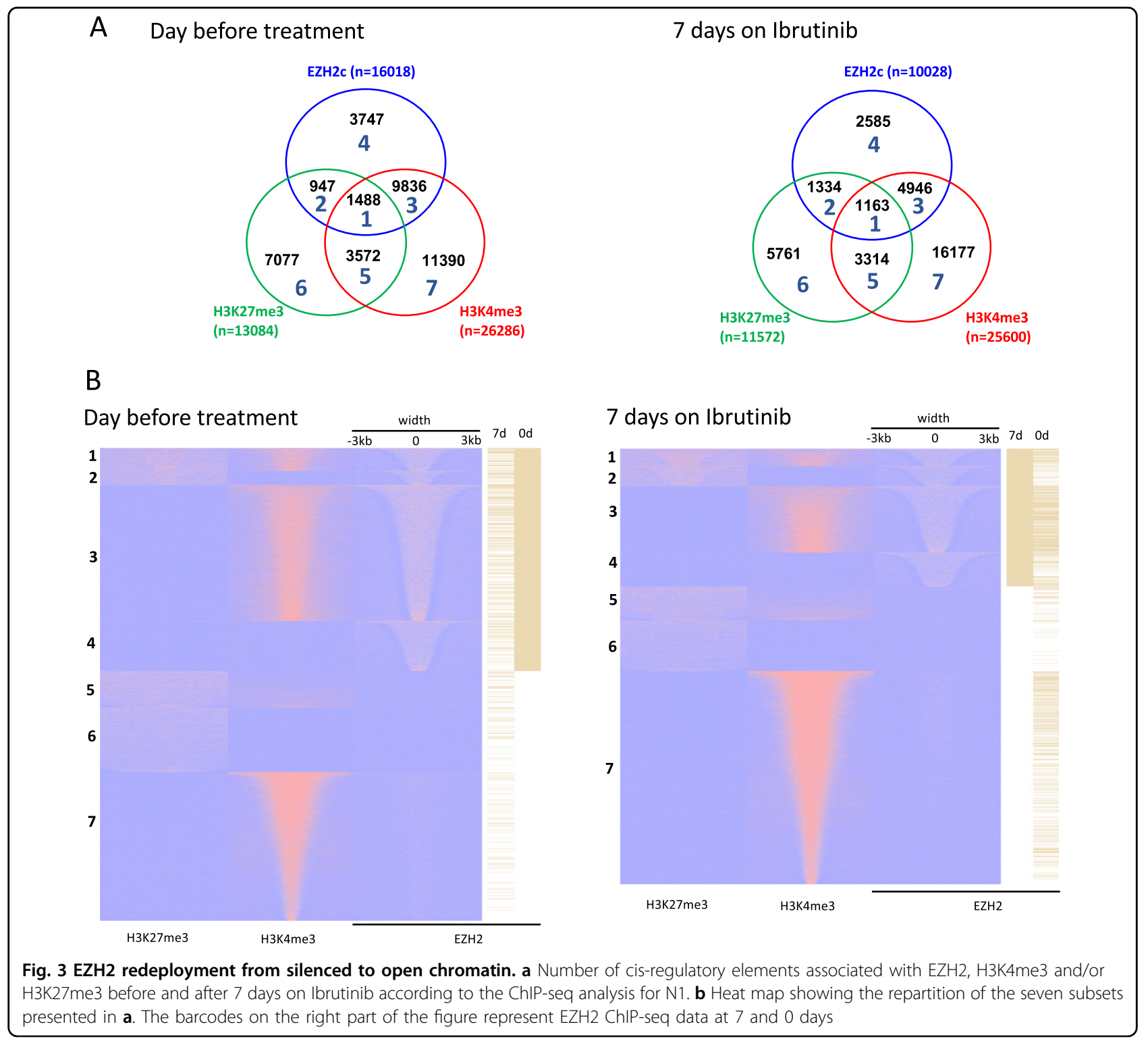

downstream genes were associated with signal transduction and response to stimuli and included FOXO1 and FOXO3, two transcription factors that mediate quiescence $^{26,27}$ (Supplementary Figs. S5A, D, E, S6A, Supplementary Table 2.1). Moreover, only two out of six samples of CLL cells showed a significant CXCR4 and RAC1 increased mRNA level in response to both TGFß (SMADactivating signal) and WNT-signalling activation in vitro (Supplementary Fig. S5F) as described from previous reports $^{28}$. These data suggest that CLL cells might be sensitive to TGFß and WNT signalling during very specific window(s) of their life cycle. In conclusion, our data suggest that some $\mathrm{H} 3 \mathrm{~K} 4 \mathrm{me} 3^{+}$-regulatory elements might be actively protected from this observed genome-wide PRC2 recruitment.
Coordinated changes in H3K4me3 peak repartition and intensity at selected cis-elements in Ibrutinib-treated CLL cells

To assess the evolution of the repartition of the $\mathrm{H} 3 \mathrm{~K}_{4} \mathrm{me}^{+}{ }^{+}$peaks between patients and in reponse to Ibrutinib, we generated scores for each set of data compared to the average scores obtained for the five samples analysed before treatment and used patient $\mathrm{N} 1$ before treatment as a reference profile as described (Supplementary Fig. S7, Supplementary Table 5). In this reference sample, EZH2 recruitment to active cis-elements correlated with increased scores for transcription factors enriched at non-promoter elements and slightly decreased ones for factors like EZH2 and SUZ12 compared to the average scores (Fig. 4a, Supplementary Table 5). 


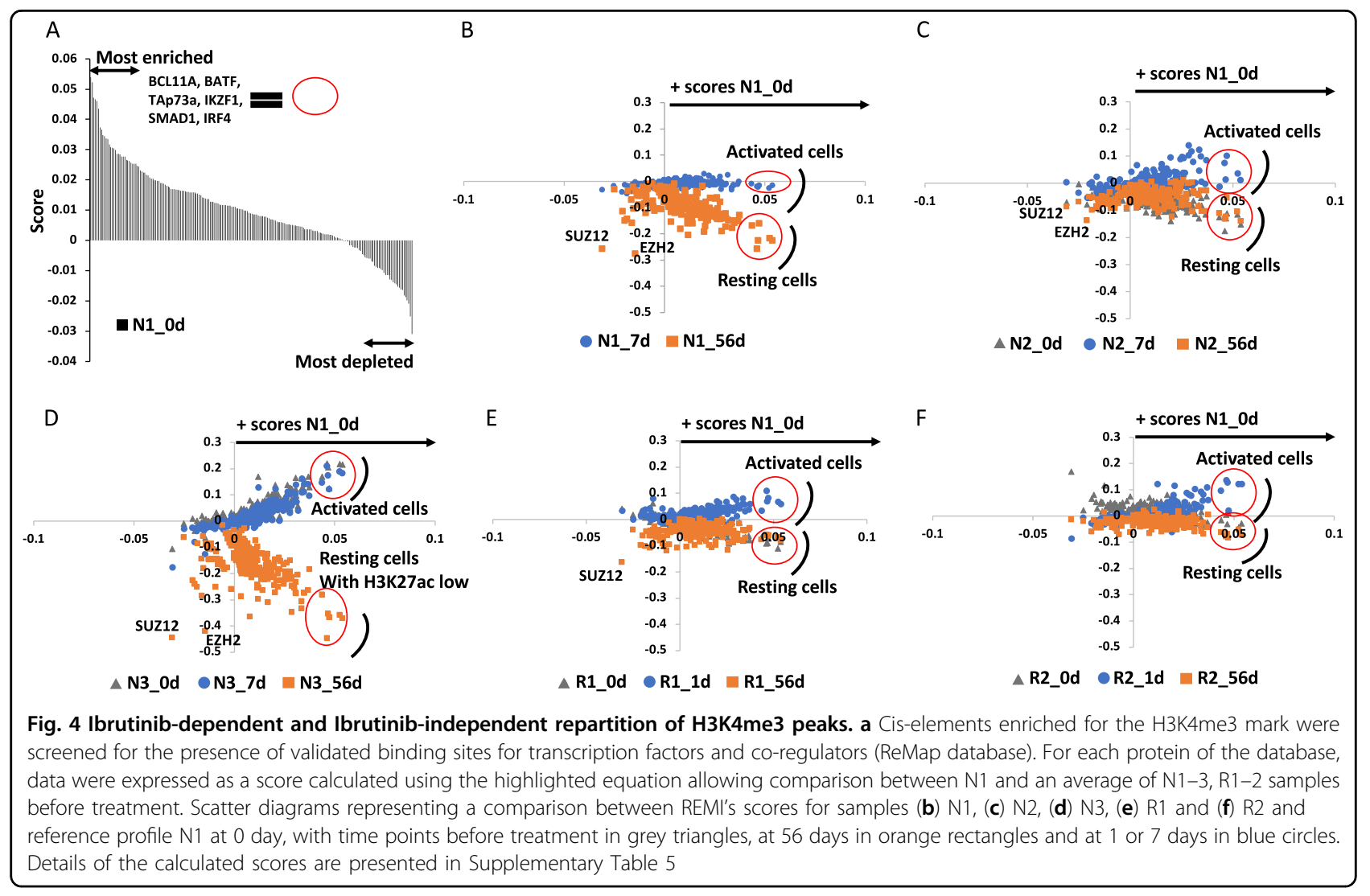

Interestingly, comparable profiles were observed for CLL cells from patients treated for a week or less with Ibrutinib (blue circles) for which the scores for transcription factors enriched at non-promoter elements are similar or even higher (Fig. 4b-f). In contrast, after 2 months treatment (orange squares), the number of H3K4me3 peaks at nonpromoter and EZH2/SUZ12-associated cis-elements were significantly reduced (Fig. 4b-f). These differences were more accentuated in TN compared to RR patients (Fig. 4f), the latter category having a prolonged phase of lymphocytosis compared to the former (Fig. 1b).

Furthermore, we identified 253 genes closest to H3K4me3 peaks found in all five blue profiles but in none of the orange ones and determined that they were coding for proteins mainly involved in cell proliferation, response to external stimulus and metabolic process (Supplementary Table 5) suggesting that these peaks were charactersitic of activated cells coming from the proliferation centre of the lymph node (Fig. $4 \mathrm{~b}-\mathrm{f}$ ). Interestingly, this family of peaks could be found before treatment in N1 and N3 but not in N2, R1 and R2 (Fig. 4b-f) suggesting episodes of lymphocytosis prior treatment.

Having analysed H3K4me3 peak repartition evolution, we then focussed on the evolution of their intensity with time on Ibrutinib. H3K4me3 global intensity stayed stable with time during treatment (Fig. 2d), but taken individually, H3K4me3 peak intensity showed variation at 1 and 56 days on Ibrutinib compared to before treatment (Fig. 5a, b). To determine if these variations were purely stochastic or were co-ordinately affecting specific families of peaks, we compared the composition of the bottom (most decreased compared with before treatment) and top (most increased compared with before treatment) peaks at 56 days for the promoter-associated and non-promoterassociated H3K4me3 marks, respectively (black boxes), with the one obtained for the total amount of H3K4me3 peaks (Fig. 5c). In agreement with changes observed for the repartition of H3K4me3 peaks, the "top" subset was enriched for transcription factors associated with activated B cells and B cell functions (Fig. 5a-c, Supplementary Table 2.2), and the "bottom" subset enriched for promoter regions associated with EZH2/SUZ12, which were identified in patient $\mathrm{N} 1$ at bivalent promoters and CpG islands (Fig. 5a-c, Supplementary Table 2.3). CtBP2, which was enriched in the "bottom" subset is not expressed in CLL but has been shown to participate in PRC2 recruitment at active embryonic stem cells during exit from pluripotency ${ }^{29}$, which reinforces the view that EZH2/SUZ12 ReMap libraries were biased toward earlier differentiation stages.

We analysed these variations in H3K4me3 peak intensity for patients N1-3 and R1-2 over time (Figs. 5 and 6, 

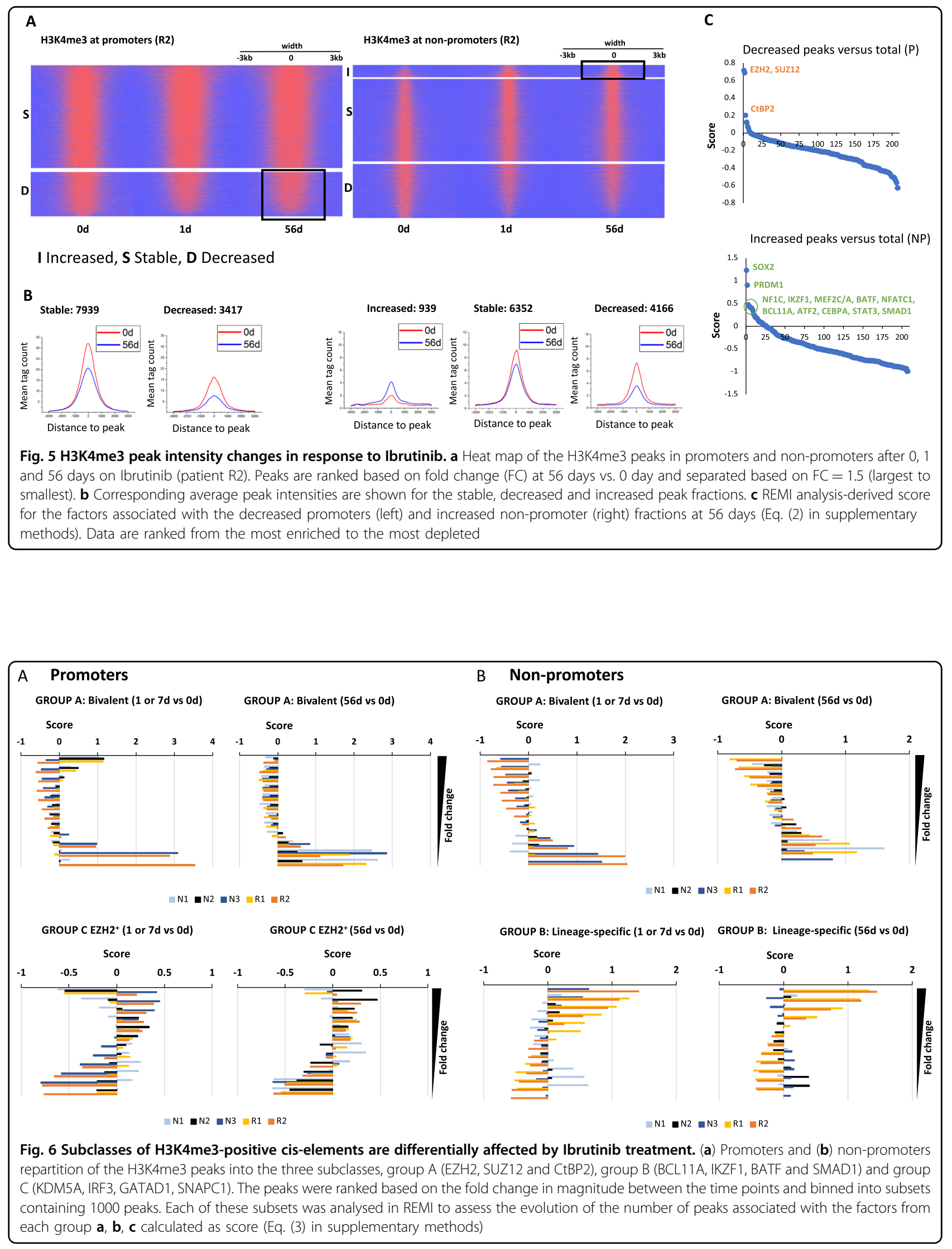
Supplementary Fig. S8 and 9). First, the heatmap profiles suggest an important heterogeneity in the amount and intensity of peak changes in response to treatment (Fig. 5a, Supplementary Fig. S8). However, when the ranked peaks (based on fold change) were binned into 1000 peak subsets and the composition of each subset analysed in REMI compared to the composition observed for all the peaks together, we identified four categories of ciselements behaving co-ordinately. Groups A and B represent the family of peaks enriched in the "bottom" subset (EZH2/SUZ12/CtBP2-containing sequences, bivalent ciselements) or in the "top" subset (IKZF1, SMAD1, BATF and BCL11A-containing sequences, lineage-specific) for patient R2, respectively (Fig. 5c, Supplementary Fig. S4). Group C (GATAD1, KDM5A, IRF3 and SNAPC1) and D (SMAD2-4) contains transcriptions factors enriched and depleted in the H3K4me3+/EZH2+ subset, respectively (Supplementary Fig. S5A). The peaks from group D were evenly distributed suggesting that these regions were not sensitive to Ibrutinib treatment (Supplementary Fig. S9A). In contrast, group A was found correlated with peaks for which H3K4me3 enrichment was decreasing in promoter and non-promoter regions after 2 months on Ibrutinib (Fig. 6a, b). These regions were mainly bivalent regions (Supplementary Fig. S9B). This effect was observed as early as 1 day for patient R2 or 7 days for patient N3 suggesting variable dynamics in response to treatment but with a common outcome (Fig. 6a, b). Group C was globally depleted from the regions enriched for group A suggesting that these peaks were protected from demethylation indicating that EZH2-recruitment was not followed by epigenetic silencing (Fig. 6a). In group B, patients $\mathrm{N} 1$ and $\mathrm{N} 2$ showed opposite distribution at 7 days (Fig. 6b) as an indication that H3K4me3 intensity was increasing at enhancer elements targeted by EZH2 (Fig. 2b, Supplementary Fig. S1). For this group, the peak repartition was the same for patients N2, N3, R1 and R2 at early time points suggesting that EZH2-transient recruitment occurred in response to Ibrutinib in four out of the five studied CLL patients (Fig. 6b). Moreover, this enrichment for "lineage specific" peaks in H3K4me3increased fraction was maintained at 56 days in both RR patients but not in TNs suggesting a difference in the kinetics of Ibrutinib response between these two categories of patients as indicated by the evolution of CLL cell count and H3K4me3 peak repartition (Figs. 1a and 4).

\section{RR CLL cells may have undertaken partial reprogramming in response to $\mathrm{FCR}$}

To further investigate the differences between the two RR and three TN patients, we selected the $825 \mathrm{RR}$-specific peaks (Fig. 7a). EZH2 and SUZ12 were the only two factors enriched in this subset suggesting activation of cisregulatory elements silenced in CLL cells from TN patients (Fig. 7a, Supplementary Fig. S10A-B). Accordingly, these polycomb-associated regions were enriched for genes involved in cell signalling and system development including functions associated with other lineages usually silenced in B cells (Supplementary Fig. S11, Supplementary Table 2.4). This analysis, restricted to "presence or absence" of H3K4me3 peaks, underestimated the differences between RR and TN patients. This is illustrated by the TIAM1 gene locus, where at the promoter H3K4me3 is low but not completely absent for N1, N2 and N3 (Supplementary Fig. S12). Interestingly, these epigenetic differences were not seen at TIAM1 mRNA level which was low in both RR and TN patients before treatment (data not shown).

\section{Discussion}

In CLL, clonal evolution leading to resistance to Ibrutinib has been described ${ }^{9}$, but the lymphocyte count stabilisation after long-term exposure to the drug observed in the majority of Ibrutinib-treated patients cannot be explained solely by the presence of resistant clones ${ }^{30,31}$. This absence of remission correlates with the epigenetic changes we observed, suggesting that cancer cells can also change phenotypically in response to treatment (Fig. 7b). Ibrutinib treatment includes loss of both H3K27ac and H3K27me3 marks in every patient tested. Hypomethylation is a characteristic of quiescent cells and an indicator of enhanced plasticity ${ }^{32-34}$. These changes could participate in the mechanisms of resistance as described previously $^{35}$.

Analysing the dynamics of lymphocytosis following Ibrutinib treatment in CLL cells reveals that the response to Ibrutinib tends to be quicker in $\mathrm{TN}$ patients and delayed in RR patients ${ }^{36}$. In this study, this delay can be linked with a significant number of promoters showing bivalency in RR patients but fully silent in TN patients, including TIAM1, which has been shown to mediate chemoresistance to fludarabine in $\mathrm{CLL}^{37}$. Maintenance of H3K4me3 at PRC2 targets in RR patients argues for a mechanism of partial reprogramming that takes place during the process of relapse induced by FCR and for which these patients have kept an epigenetic memory which could decrease their sensitivity to Ibrutinib. Further investigation with more CLL samples will be necessary to confirm this point.

EZH2 expression is restricted to proliferative cells in cancer and non-transformed human cells ${ }^{38}$ and, accordingly, to the proliferation centre in $\mathrm{B}$ cells ${ }^{18,19}$. Therefore, transient increases in protein level as well as an enhanced interaction with chromatin, and more specifically with $\mathrm{H} 3 \mathrm{~K} 4 \mathrm{me}^{+}{ }^{+}$cis-elements in correlation with treatment initiation, can be interpreted by an enrichment of CLL cells accumulating in the blood from the lymph node. Interestingly, a significant level of EZH2 protein and 


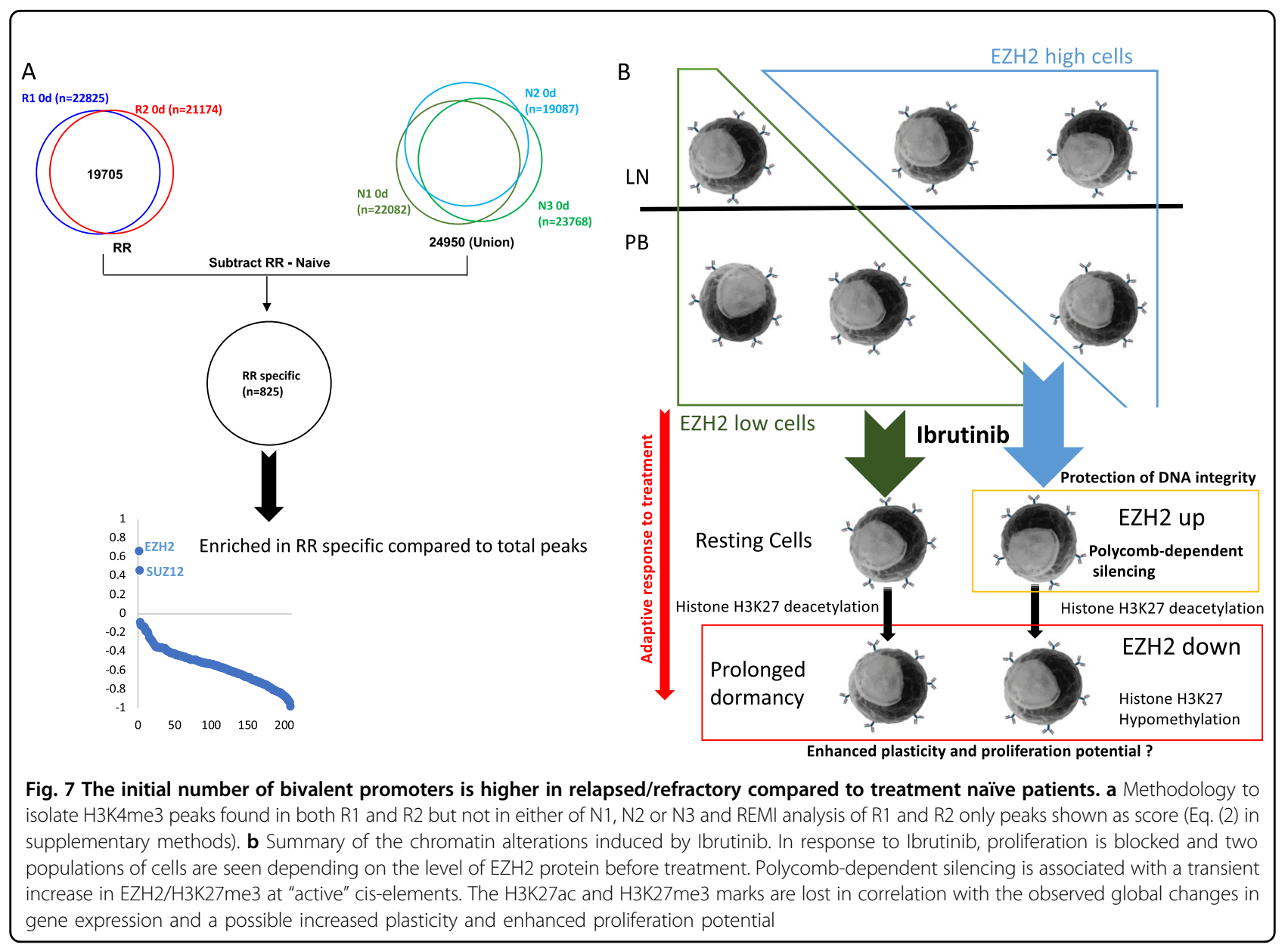

EZH2-chromatin association on the day of treatment initiation could also be seen as an indication of a drugindependent mechanism of lymphocytosis commonly seen in CLL. When EZH2 was measured at day 0, treatment initiation was not provoking any surge in EZH2expressing cells to the $\mathrm{PB}$ suggesting temporary exhaustion of these cells in the lymph node post lymphocytosis. Moreover, H3K27me3 increase at EZH2-bound cis-elements was relatively subtle when EZH2 was associated with chromatin at the beginning of the treatment suggesting that EZH2 might be recruited in an inactivated form. Transcription can repress PRC2 activity through its interaction with nascent transcripts ${ }^{39,40}$ and therefore antagonising PRC2 binding to chromatin $^{41}$. Our data suggest a possible two-step mechanism in which PRC2 is recruited to active cis-elements preventing promoter-enhancer interaction. At this stage, a low level of transcription might prevent $\mathrm{PRC} 2$ interaction with chromatin and H3K27 acetylation/methylation switch. Then in response to Ibrutinib, additional repression mechanisms may play a role in favour of a strong repressive state and H3K27me3 deposition. A similar mechanism has been described in Drosophila in response to heat shock accompanied by a global chromatin 3D reorganisation driven by polycomb ${ }^{42}$.

In addition, an in-depth analysis of the evolution of H3K4me3 peak repartition and intensity during the first 2 months of treatment revealed epigenomic plasticity both Ibrutinib-dependent and Ibrutinib-independent. First, regions targeted by EZH2 were protected from H3K4me3 demethylation and, for non-promoter elements targeted by B-cell-specific transcription factors, correlated with a transient increase in H3K4me3. This may be due to an increase in enhancer-specific noncoding RNA (eRNA) transcription. Second, stable bivalent cis-elements appeared to be among the regions excluded from this transient EZH2 recruitment, which was in agreement with a role for EZH2 in de novo H3K27 methylation and EZH1 for the maintenance of this mark $^{43}$. Moreover, these bivalent cis-elements showed a trend toward reduction of H3K4me3 peak intensity in all patients, suggesting a correlation between a prolonged exit from the cell cycle as a consequence of Ibrutinib treatment and demethylation of both H3K27me3 and H3K4me3 at silenced chromatin.

Some H3K4me3+ cis-elements were specifically protected from EZH2 recruitment and in particular regions 
potentially targeted by SMAD2-4. TGFß has been shown to be inhibitory at almost any B-cell stage ${ }^{44}$ and may participate in the reorganisation of the transcriptome during the transition from the proliferative to the resting phase. This hypothesis is reinforced by the presence of FOXO1 and FOXO3 in the list of genes protected from EZH2 recruitment and potentially targeted by SMAD2-4. FOXO1 and FOXO3 control a transcriptional programme repressing cell proliferation and promoting resistance to stress and survival ${ }^{45}$. Moreover, the expression of these two transcription factors is downregulated by the $\mathrm{BCR}$ signalling ${ }^{46}$. Interestingly, SMAD6 and SMAD7, the two inhibitors of TGFß signalling ${ }^{47,48}$ are also among the genes identified within the EZH2 ${ }^{-} / \mathrm{SMAD} 2-4^{+}$fraction, arguing for a short window of activation of this signal as suggested by the observed variability in CLL response to TGFß1 in vitro. In addition, TGFß-induced quiescence has been recently shown to mediate chemoresistance in squamous cell carcinoma ${ }^{49}$. Altogether, these data suggest that the passage from proliferating to quiescence in response to Ibrutinib is controlled by polycomb and potentially TGFß.

In conclusion, chromatin structure changes dynamically in response to Ibrutinib in CLL cells in a two-step mechanism leading to the loss of H3K27 marks and correlated with the stabilisation of CLL cell count. Histone hypomethylation associated with prolonged exit from the cell cycle has been connected to mechanisms of survival and enhanced plasticity and can be seen as an adaptive response to Ibrutinib. Further investigation will be necessary to assess if these changes provoke a reduced sensitivity to treatment and participate in the mechanism of relapse.

\section{Materials and methods Isolation of CLL cells}

Peripheral blood was collected from patients with informed written consent (St. James' University Hospital, approval number 14/YH/0034). Peripheral blood mononuclear cells (PMBCs) were isolated by density gradient centrifugation using Lymphoprep ${ }^{\mathrm{TM}}$ (Axis-Shield). CLL cells were further purified using CD19+ MicroBeads (Miltenyi Biotec). Due to the restricted cell number for each collected time points, purified cells were used for immunoblotting, gene expression or chromatin immunoprecipitation as described below.

\section{Immunoblot analysis}

Cells were lysed in a buffer containing $50 \mathrm{mM}$ Tris- $\mathrm{HCl}$ $(\mathrm{pH}$ 6.8), 1.5\% SDS and 1x protease inhibitor cocktail (ThermoFischer Scientific). Protein concentration was determined using Pierce $\mathrm{BCA}^{\mathrm{Tw}}$ Protein Assay (ThermoFischer Scientific). Protein from each cell-lysate was separated by SDS-PAGE, followed by electrotransfer into a nitrocellulose membrane. Proteins of interest were visualised and quantified after primary antibody incubation using Pierce ${ }^{\mathrm{Tx}}$ ECL Western Blotting Substrate (ThermoFisher Scientific) and ChemiDoc Imaging System (Bio-Rad). Mean band intensity was calculated using Image Lab software. Primary antibodies were as follows: H3K4me3 (Millipore; 04-745), H3K27me3 (Millipore; 07449), H3K27ac (Millipore; 07-360) and EZH2 (Diagenode; pAb-039-050) were used as primary antibodies; anti$\beta$-actin (Sigma; A1978) and anti-Total H3 (Abcam; ab1791) were used as loading controls.

\section{Chromatin immunoprecipitation (ChIP) and ChIP-seq}

ChIP was performed as previously described ${ }^{50,51}$ using dynabeads protein $\mathrm{G}$ (Invitrogen) with $2.4 \mathrm{mg}$ per $10 \mathrm{ml}$ beads with $1 \mu \mathrm{g}$ anti-H3K4me3 (Millipore; 04-745), antiH3K27ac (Millipore; 07-360), anti-H3K27me3 (Millipore; 07-449) and anti-EZH2 (Diagenode; pAb-039-050) antibodies. See Supplementary Table 3 for primer sequences.

For sequencing (ChIP-seq), libraries were prepared using NEBNext ${ }^{\circ}$ Ultra $^{\text {Tn }}$ II DNA Library Prep Kit (New England Biosciences). See Supplementary Methods for sequencing run details and Supplementary 4 for QC of these libraries.

\section{Bioinformatics analysis}

See Supplementary Methods for further details. Sequence data from this study have been submitted to ArrayExpress (https://www.ebi.ac.uk/arrayexpress/) under accession number E-MTAB-6410.

\section{Acknowledgements \\ This work was supported by Bloodwise trial associated research project (14016). The authors thank Remi Robert who designed the ReMap and Epigenetic Mark Intersect programme and Pr. Ulf Klein for helpful discussions and criticisms.}

\section{Author details \\ 'Section of Experimental Haematology, Leeds Institute of Medical Research at St James's, University of Leeds, Leeds, UK. Bioinformatics Group, Institute of Molecular and Cellular Biology, University of Leeds, Leeds, UK. ${ }^{3}$ Haematological Malignancy Diagnostic Service (HMDS), St. James's Institute of Oncology, Bexley Wing Beckett Street, Leeds LS9 7TF, UK}

Conflict of interest

The authors declare that they have no conflict of interest.

\section{Publisher's note}

Springer Nature remains neutral with regard to jurisdictional claims in published maps and institutional affiliations.

Supplementary Information accompanies this paper at (https://doi.org/ 10.1038/s41389-019-0142-2).

Received: 22 December 2018 Accepted: 1 April 2019

Published online: 10 May 2019 


\section{References}

1. Howlader, N. N. A. et al. (eds). SEER Cancer Statistics Review, 1975-2010. (National Cancer Institute, Bethesda, MD, 2013).

2. Hallek, M. et al. Addition of rituximab to fludarabine and cyclophosphamide in patients with chronic lymphocytic leukaemia: a randomised, open-label, phase 3 trial. Lancet 376, 1164-1174 (2010).

3. Herman, S. et al. Phosphatidylinositol 3-kinase- $\delta$ inhibitor CAL-101 shows promising preclinical activity in chronic lymphocytic leukemia by antagonizing intrinsic and extrinsic cellular survival signals. Blood 116, 2078-2088 (2010).

4. Hoellenriegel, J. et al. Selective, novel spleen tyrosine kinase (Syk) inhibitors suppress chronic lymphocytic leukemia B-cell activation and migration. Leukemia 26, 1576-1583 (2012).

5. Byrd, J. C. et al. Ibrutinib versus ofatumumab in previously treated chronic lymphoid leukemia. New Engl. J. Med. 371, 213-223 (2014).

6. Burger, J. A. et al. Ibrutinib as initial therapy for patients with chronic lymphocytic leukemia. New Engl. J. Med. 373, 2425-2437 (2015).

7. Collett, L. et al. Assessment of ibrutinib plus rituximab in front-line CLL (FLAIR trial): study protocol for a phase III randomised controlled trial. Trials 18, 387 (2017).

8. Kaur, V. \& Swami, A. Ibrutinib in CLL: a focus on adverse events, resistance, and novel approaches beyond ibrutinib. Ann. Hematol. 96, 1175-1184 (2017).

9. Ahn, I. E. et al. Clonal evolution leading to ibrutinib resistance in chronic lymphocytic leukemia. Blood 129, 1469-1479 (2017).

10. Martinez, A.-M. M. \& Cavalli, G. The role of polycomb group proteins in cell cycle regulation during development. Cell Cycle 5, 1189-1197 (2006).

11. Valk-Lingbeek, M. E., Bruggeman, S. W. \& van Lohuizen, M. Stem cells and cancer; the polycomb connection. Cell 118, 409-418 (2004).

12. Sparmann, A. \& van Lohuizen, M. Polycomb silencers control cell fate, development and cancer. Nat. Rev. Cancer 6, 846-856 (2006).

13. Hosogane, M., Funayama, R., Shirota, M. \& Nakayama, K. Lack of transcription triggers H3K27me3 accumulation in the gene body. Cell Rep. 16, 696-706 (2016).

14. Bernstein, B. E. et al. A bivalent chromatin structure marks key developmental genes in embryonic stem cells. Cell 125, 315-326 (2006).

15. Béguelin, W. et al. EZH2 is required for germinal center formation and somatic EZH2 mutations promote lymphoid transformation. Cancer Cell 23, 677-692 (2013).

16. Wassef, M. et al. Impaired PRC2 activity promotes transcriptional instability and favors breast tumorigenesis. Genes Dev. 29, 2547-2562 (2015).

17. Su, I. H. H. et al. Ezh2 controls B cell development through histone H3 methylation and Igh rearrangement. Nat. Immunol. 4, 124-131 (2003).

18. van Galen, J. C. et al. Distinct expression patterns of polycomb oncoproteins and their binding partners during the germinal center reaction. Eur. J. Immunol. 34, 1870-1881 (2004).

19. Velichutina, I., Shaknovich, R., Geng, H. \& Blood, J.-N. A. EZH2-mediated epigenetic silencing in germinal center $B$ cells contributes to proliferation and lymphomagenesis. Blood 116, 5247-5255 (2010).

20. Beekman, R. et al. The reference epigenome and regulatory chromatin landscape of chronic lymphocytic leukemia. Nat. Med. 24, 868-880 (2018).

21. de Rooij, M. F. et al. The clinically active BTK inhibitor PCl-32765 targets B-cell receptor- and chemokine-controlled adhesion and migration in chronic lymphocytic leukemia. Blood 119, 2590-2594 (2012).

22. $\mathrm{Yu}, \mathrm{Y}$. et al. Bcl11a is essential for lymphoid development and negatively regulates p53. J. Exp. Med. 209, 2467-2483 (2012).

23. Murphy, T. L., Tussiwand, R. \& Murphy, K. M. Specificity through cooperation: BATF-IRF interactions control immune-regulatory networks. Nat. Rev. Immunol. 13, 499-509 (2013).

24. Schwickert, T. A. et al. Stage-specific control of early B cell development by the transcription factor Ikaros. Nat. Immunol. 15, 283-293 (2014).

25. Bollum, L. K. et al. BMP-7 induces apoptosis in human germinal center B cells and is influenced by TGF- $\beta$ receptor type I ALK5. PLOS ONE 12, e0177188 (2017).

26. Fernandez, P. C. et al. Genomic targets of the human c-Myc protein. Genes Dev. 17, 1115-1129 (2003).

27. Kops, G. J. et al. Forkhead transcription factor FOXO3a protects quiescent cells from oxidative stress. Nature 419, 316-321 (2002).
28. Lotz, M., Ranheim, E. \& Kipps, T. J. Transforming growth factor beta as endogenous growth inhibitor of chronic lymphocytic leukemia B cells. J. Exp. Med. 179, 999-1004 (1994).

29. Kim, T. W. et al. Ctbp2 modulates NuRD-mediated deacetylation of H3K27 and facilitates PRC2-mediated H3K27me3 in active embryonic stem cell genes during exit from pluripotency. Stem Cells 33, 2442-2455 (2015).

30. Byrd, J. C. et al. Targeting BTK with ibrutinib in relapsed chronic lymphocytic leukemia. New Engl. J. Med. 369, 32-42 (2013).

31. Komarova, N. L., Burger, J. A. \& Wodarz, D. Evolution of ibrutinib resistance in chronic lymphocytic leukemia (CLL). Proc. Natl. Acad. Sci. USA 111 13906-13911 (2014)

32. Baxter, J. et al. Histone hypomethylation is an indicator of epigenetic plasticity in quiescent lymphocytes. EMBO J. 23, 4462-4472 (2004).

33. Kallingappa, P. K. et al. Quiescence loosens epigenetic constraints in bovine somatic cells and improves their reprogramming into totipotency. Biol. Reprod. 95, 16 (2016)

34. Lee, J. et al. Signalling couples hair follicle stem cell quiescence with reduced histone H3 K4/K9/K27me3 for proper tissue homeostasis. Nat. Commun. 7, 11278 (2016).

35. Pisco, A. O. et al. Non-Darwinian dynamics in therapy-induced cancer drug resistance. Nat. Commun. 4, 2467 (2013).

36. Smith, D. D. et al. Modeling absolute lymphocyte counts after treatment of chronic lymphocytic leukemia with ibrutinib. Ann. Hematol. 94, 249-256 (2015).

37. Hofbauer, S. W. et al. Tiam1/Rac1 signals contribute to the proliferation and chemoresistance, but not motility, of chronic lymphocytic leukemia cells. Blood 123, 2181-2188 (2014).

38. Bracken, A. P. et al. EZH2 is downstream of the pRB-E2F pathway, essential for proliferation and amplified in cancer. EMBO J. 22, 5323-5335 (2003).

39. Kanhere, A., Viiri, K., Araújo, C. C. \& cell, R.-J. Short RNAs are transcribed from repressed polycomb target genes and interact with polycomb repressive complex-2. Mol. Cell 38, 675-688 (2010).

40. Kaneko, S., Son, J., Shen, S. S. \& structural, R. -D. PRC2 binds active promoters and contacts nascent RNAs in embryonic stem cells. Nat. Struct. 20, 1258-1264 (2013).

41. Beltran, $M$. et al. The interaction of PRC2 with RNA or chromatin is mutually antagonistic. Genome Res. 26, 896-907 (2016).

42. Li, L. et al. Widespread rearrangement of $3 D$ chromatin organization underlies polycomb-mediated stress-induced silencing. Mol. Cell 58, 216-231 (2015).

43. Son, J., Shen, S. S., Margueron, R. \& Reinberg, D. Nucleosome-binding activities within JARID2 and EZH1 regulate the function of PRC2 on chromatin. Genes Dev. 27, 2663-2677 (2013).

44. Kaminski, D. A., Letterio, J. J. \& Burrows, P. D. Differential regulation of mouse B cell development by transforming growth factor $\beta 1$. Dev. Immunol. 9, 86-95 (2002).

45. Limon, J. J. \& Fruman, D. A. Akt and mTOR in B cell activation and differentiation. Front. Immunol. 3, 228 (2012)

46. Yusuf, I., Zhu, X., Kharas, M. G., Chen, J. \& Fruman, D. A. Optimal B-cell proliferation requires phosphoinositide 3-kinase-dependent inactivation of FOXO transcription factors. Blood 104, 784-787 (2004).

47. Imamura, T. et al. Smad6 inhibits signalling by the TGF- $\beta$ superfamily. Nature 389, 39355 (1997)

48. Hayashi, $\mathrm{H}$. et al. The MAD-related protein Smad7 associates with the TGFbeta receptor and functions as an antagonist of TGFbeta signaling. Cell $\mathbf{8 9}$ 1165-1173 (1997).

49. Brown, J. A. et al. TGF- $\beta$-induced quiescence mediates chemoresistance of tumor-propagating cells in squamous cell carcinoma. Cell Stem Cell 21, 6501975490560 (2017).

50. Lefevre, P., Melnik, S., Wilson, N., Riggs, A. D. \& Bonifer, C. Developmentally regulated recruitment of transcription factors and chromatin modification activities to chicken lysozyme cis-regulatory elements in vivo. Mol. Cell. Biol. 23 4386-4400 (2003).

51. Lefevre, P., Witham, J., Lacroix, C. E., Cockerill, P. N. \& Bonifer, C. The LPS induced transcriptional upregulation of the chicken lysozyme locus involves CTCF eviction and noncoding RNA transcription. Mol. Cell 32 129-139 (2008) 